\title{
EDITORIAL
}

\section{Messenger RNA Vaccines are Safe \& Effective and do not Modify Host DNA}

Despite the fact that over 5.3 billion doses of COVID-19 vaccines have been administered globally, there still exists measurable hesitancy in a select cohort of the population to get vaccinated. This reluctance seems to be more evident towards messenger RNA (mRNA) vaccines which is attributed largely to misinformation about their safety and efficacy and the unfounded fear that they may integrate into host DNA thus modifying it permanently. In this editorial, we plan to provide scientific evidence to repudiate these misconceptions and to alleviate concerns related to COVID-19 mRNA vaccines.

On December 11, 2020, US Food \& Drug Administration (FDA) issued its first Emergency Use Authorization (EUA) for Pfizer/BioNTech COVID-19 vaccine for the prevention of COVID19 caused by SARS-CoV-2 in individuals 16 years of age and over (1). The Pfizer/BioNTech vaccine is a nucleoside-modified mRNA encoding for the viral spike glycoprotein of SARS-CoV-2 formulated in lipid particle (1). On May 10, 2021, FDA issued another EUA approving the use of Pfizer/BioNTech vaccine for prevention of COVID-19 in individuals 12-15 years of age (2). Given the high risk of infection with the Delta variant of vaccinated individuals with immunosuppressed status, on August 12, 2021, FDA modified its EUA allowing Pfizer/BioNTech vaccine to be administered as a third dose to this select cohort of patients.

With the availability of additional safety and efficacy data from millions of people who have been vaccinated supplemented by that obtained from double-blinded placebo-controlled clinical trials, Pfizer submitted a Biologics License Application (BLA) with FDA requesting full approval of the Pfizer/BioNTech vaccine for use in individuals 12 years of age and older. After careful review, FDA approved Pfizer's BLA on August 23, 2021, making it the first and only vaccine for prevention of infection with SARS-CoV-2 (3).
Pfizer/BioNTech vaccine is now marketed under the generic name of Comirnaty.

Subsequently, on December 18, 2020, FDA issued another EUA for Moderna COVID-19 vaccine for the prevention of COVID-19 caused by SARSCoV-2 in individuals 18 years of age and over (4). The formulation of Moderna's mRNA vaccine is very similar to Pfizer/BioNTech vaccine and was recommended at a dosing regimen of two doses administered one month apart. Moderna mRNA vaccine was also approved by the FDA for a third dose in select vaccinated immunocompromised individuals who are at high risk of developing infection with SARS-CoV-2.

The process outlined above underscores the scrupulous details with which, the Federal regulatory agencies review and approve EUA's and BLA's. This process is stringently coordinated by various independent oversight committees including but not limited to the Advisory Committee on Immunization Practices - an entity within the US Centers for Disease Control \& Prevention that is composed of experts in the field of infectious diseases and immunology and is responsible for providing guidance for the most effective control of diseases that can be prevented using vaccines.

Both Pfizer/BioNTech and Moderna vaccines are strands of messenger ribonucleic acid (mRNA) encapsulated in lipid nanoparticles. The mRNA encodes for the antigenic glycoproteins of the Spike (S) domain of SARS-CoV-2 with two proline substitutions (K986P and V987P mutations) which, stabilizes the perfusion conformation of the glycoprotein. Once delivered by intramuscular administration, the lipid nanoparticles facilitate the uptake of the mRNA vaccine by the host antigen presenting cells (such as dendritic cells or macrophages) and its entry into the cytosol (the part of the cell that is outside the nucleus which houses the DNA) [Figure 1]]. Utilizing the host cell's 
ribosomes (organelle that help in protein synthesis), the mRNA vaccine is translated into Spike proteins which are presented on the cell surface to raise $T$ and $\mathrm{B}$ cell responses. The mRNA vaccine in the cytosol is degraded by the cell in a few days whereas, similar to other proteins made by the body, the Spike protein survives for a few weeks and is ultimately cleared from the system.

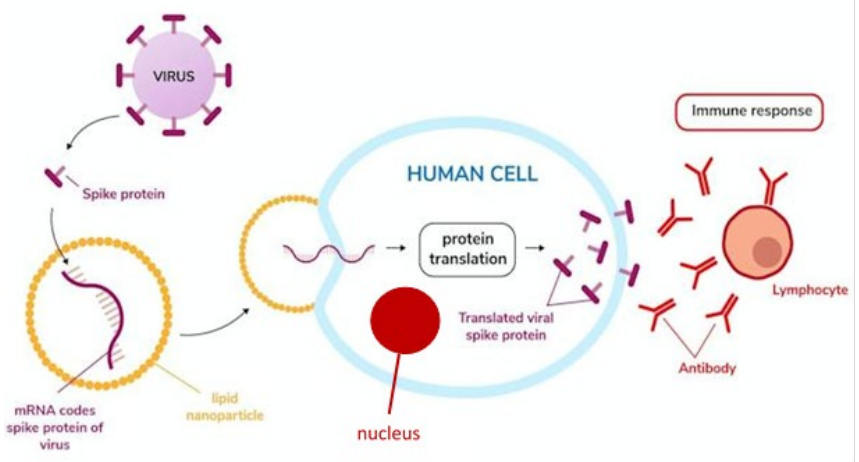

Figure 1: The normal "physiological" pathway utilized by the COVID-19 mRNA vaccines to produce Spike proteins that raise both cell and antibody mediated immune responses.

In conclusion, mRNA-based vaccines are safe, effective, and capable of raising both antibodymediated and cellular immune responses against SARS-CoV-2. Additionally, all available scientific data provides unequivocal evidence that mRNAbased vaccines follow the normal physiological pathways and once they enter, they remain confined to the extra-nuclear part of the host cell and are not designed to gain entry into the nucleus and are therefore incapable of modifying the DNA. Gaining public confidence is paramount in the success of the ongoing vaccination efforts to mitigate the spread of COVID-19. Therefore, every effort must be made to discount the misinformation and provide to the community facts that are informed by strong scientific evidence.

\section{References}

1. US Food and Drug Administration's Letter of Emergency Use Authorization for Pfizer/BioNTech mRNA Vaccine for individuals 17 years of age and older. December 11, 2020, https://nps.edu/documents/111291366/124403968/PfizerBioNTech+COVID-

19+Vaccine+EUA+LOA+\%2811+Dec+2020\%29.pdf/106e 966 2-956c-aa94-8d4b-a945a6a10b87?t=1608071313236
2. US Food and Drug Administration's Letter of Emergency Use Authorization for Pfizer/BioNTech mRNA Vaccine for individuals 12-16 years of age. May 10, 2021. https://www.fda.gov/media/144412/download

3. US Food and Drug Administration's Letter of Approval of Biologics License Application for Pfizer/BioNTech vaccine. August 23, 2021. https://www.fda.gov/media/151710/download

4. US Food and Drug Administration's Letter of Emergency Use Authorization for Moderna mRNA Vaccine. December 18, 2020. content/uploads/2020/12/Moderna COVID19 Vaccine EUA Letter of Authorization.pdf

\section{Sohail Rao, MD, MA, DPhil}

Executive Vice President, DHR Health, 5501 S. McColl Road, Edinburg, Texas

President \& Chief Executive Officer, DHR Health Institute for Research \& Development, 5323 S. McColl Road, Edinburg, Texas

Corresponding author email: s.rao@dhr-rgv.com

\section{Disclosures: None}

ORCID: Sohail Rao: https://orcid.org/0000-0001-5027$\underline{9992}$

\section{Manish Singh, MD, FACS}

Chief Executive Officer, DHR Health, 5501 S. McColl Road, Edinburg, Texas

Disclosures: None

ORCID: Manish Singh: https://orcid.org/0000-00034146-3282 